REPERTORIO 



\title{
TRIBUNAL EUROPEO DE DERECHOS HUMANOS: MUTACIONES Y DESAFÍOS. REPERTORIO BIBLIOGRÁFICO $(2007-2018)^{1}$
}

\author{
LUCÍA ALONSO SANZ \\ Profesora ayudante doctora de Derecho Constitucional \\ Universidad Loyola Andalucía
}

SUMARIO

\begin{abstract}
Nota Preliminar. I. Organización y procedimientos. II. Sentencias: contenido y efectos. Ejecución y supervisión. Medidas provisionales. III. Principio de subsidiariedad, margen de apreciación nacional y consenso europeo. IV. Diálogo jurisprudencial. V. Revistas jurídicas que incluyen periódicamente crónicas de jurisprudencia del TEDH.
\end{abstract}

\section{NOTA PRELIMINAR}

El presente repertorio, integrado en el monográfico sobre el Tribunal Europeo de Derechos Humanos, ofrece una selección de obras sobre la institución publicadas desde hace algo más de una década.

Una recopilación de bibliografía sobre el TEDH es un objeto inmenso y hemos debido acotar su extensión adoptando algunas convenciones. Nos ocuparemos de la institución como tal: organización, funcionamiento, impacto de su actuación, diálogo e interacción con otras instituciones. Así como de ciertos criterios que orientan —o así lo pretenden- el alcance de su actuación frente a la de los Estados parte en el Convenio Europeo de Derechos Humanos, intentando arrojar luz sobre esa delicada frontera de legitimidades, a saber, el principio de subsidiariedad y los conceptos de consenso y de margen de apreciación nacional. Salvo este último punto, que se considera desde un prisma trasversal, como un parámetro de actuación del Tribunal más que como un elemento de decisión de conflictos concretos, no se extiende la recopilación a la dimensión material del fondo de las resoluciones. Quedan excluidas, por tanto, las obras que analizan la jurisprudencia del Tribunal, ya sea sobre casos concretos, o sobre un determinado problema o derecho o en relación a con un determinado Estado (o Estados), excepto cuando se trate de España y siempre que ataña a una de las cuestiones generales objeto de la recopilación. Queda fuera de la misma, por último, un asunto que consideramos que depende más de la Unión Europea que del propio Tribunal, cual es el del azaroso proceso de adhesión de aquélla al Convenio.

1 La autora agradece las valiosas aportaciones de la Dra. Carmen Morte-Gómez, Letrada-Jefa de División del Tribunal Europeo de Derechos Humanos, en la elaboración de este trabajo, así como la labor de revisión realizada por el Prof. Javier García Roca. 
En cuanto a la metodología utilizada, se han tomado como base para la selección de las obras los catálogos virtuales de las bibliotecas del propio Tribunal de Estrasburgo y del Centro de Estudios Políticos y Constitucionales, así como la base de datos Dialnet.

En relación a los criterios lingüísticos, la naturaleza supranacional del objeto del repertorio imponía que englobara doctrina extranjera; pero sería imposible hacerlo con todas las lengua europeas. De ahí que finalmente se optara por incluir, además de obras en castellano, trabajos escritos en inglés y, en menor medida, en francés, los dos idiomas oficiales del Tribunal.

Respecto al periodo temporal cubierto por la recopilación, dado el constante proceso de cambio en que el Tribunal está imbuido en los últimos tiempos, que explica que muchos de los estudios sobre la materia se realicen en clave diacrónica; y teniendo en cuenta que lo que se pretende en este trabajo, como se precisará a continuación, es sobre todo mostrar los desafíos actuales de la institución, se ha optado por acotar la selección a las obras publicadas desde el año 2007 (incluido).

La definición de las distintas secciones en las que se estructura este trabajo se ha realizado $a$ posteriori del primer rastreo bibliográfico y en vistas a los resultados por él arrojados, siguiendo un método inductivo y no otro deductivo y principialista. De manera que, más que una definición, tal delimitación temática es el reflejo de los enclaves dogmáticos que más inquietan e interesan a la doctrina. Ello obedece a la finalidad principal de esta recopilación, que no es otra que ofrecer una panorámica sobre los principales problemas y retos a los que se enfrenta el TEDH. De ahí que, además de los temas excluidos en virtud de los criterios de selección precitados (que entendemos pueden distorsionar en cierta medida el retrato), haya otros que hayan quedado fuera por no concitar actualmente un interés doctrinal mayoritario.

Por último, el repertorio cierra con la referencia a algunas revistas que incluyen periódicamente crónicas jurisprudenciales o comentarios doctrinales sobre la jurisprudencia del Tribunal Europeo de Derechos Humanos.

\section{ORGANIZACIÓN Y PROCEDIMIENTOS}

\section{Libros y capítulos de libros}

BARKhUYSEN, T. and VAN EMMERIK, M.: «Legitimacy of European Court of Human Rights judgments: procedural aspects», Nick Huls, N.; AdAms, M., and BomHoff, J.: The legitimacy of highest courts' rulings: judicial deliberations and beyond, T.M.C. Asser Press, The Hague, 2009, pp. 437-449.

Benoît-Rohmer, F.: «Le protocole 16 à la Convention européenne des droits de l'homme: du soliloque au dialogue», JACQUÉ, J.P. (et alii): Ecrits sur la communauté internationale: enjeux juridiques, politiques et dimplomatiques (i.e. diplomatiques): liber amicorum Stelios Perrakis $=$ On the international community: legal, political, diplomatic issues: liber amicorum Stelios Perrakis, I. Sideris, Athens, 2017, pp. 431-446.

Besson, S. (ed.): La Cour européenne des droits de l'bomme après le Protocole 14: premier bilan et perspectives $=$ The European Court of Human Rights after Protocol 14: preliminary assessment and perspectives, Schulthess Médias Juridiques, Genève; Zürich; Bâle, 2011.

BJörgvinsson, D. T.: «The «pilot-judgment» procedure of the European Court of Human Rights», Alfredsson, G. (ed.): International human rights monitoring mechanisms: essays in honour of Jakob Th. Möller, Martinus Nijhoff Publishers, Leiden; Boston, 2009, pp. 529-540.

Bultrini, A.: The future of the European Convention on Human Rights after the Brighton Conference, Istituto Affari Internazionali, Roma, 2012.

BüRLI, N.: Third-party interventions before the European Court of Human Rights: amicus curiae, member-State and third-party interventions, Intersentia, Cambridge; Antwerp; Portland, 2017. 
BuYse, A.: «Flying or landing? The pilot judgment procedure in the changing European human rights architecture», MJöll ARnARdótTIR, O. and BuYse, A (ed.): Shifting centres of gravity in buman rights protection: retbinking relations between the ECHR, EU and national legal orders, Routledge, Abingdon; Oxon; New York, 2016, pp. 101-115.

Cano-Palomares, G.: «El procedimiento ante el Tribunal Europeo de Derechos Humanos: principales novedades desde la entrada en vigor del Protocolo n. ${ }^{\circ} 14$ al CEDH», Queralt Jiménez, A. (coord.): El Tribunal de Estrasburgo en el espacio judicial europeo; Thomas Reuters Aranzadi, Cizur Menor, 2013, pp. 27-50.

Caflisch, L.: «La Déclaration d'Interlaken: un tournant dans l'histoire de la Cour de Strasbourg?», Titiun, P. (coord.): La conscience des droits: mélanges en l'honneur de Jean-Paul Costa, Dalloz, Paris, 2011, pp. 95-108.

Council of Europe. STEERING CommitTeE For Human Rights: CDDH final report on measures that result from the Interlaken Declaration that do not require amendment of the European Convention on Human Rights, Council of Europe, Strasbourg, 2010.

- Directorate General of Human Rights and Rule of Law: Reforming the European Convention on Human Rights: Interlaken, İmir, Brighton and beyond: a compilation of instruments and texts relating to the ongoing reform of the ECHR, Council of Europe, Strasbourg, $2014^{2}$.

- Directorate General of Human Rights and Legal Affairs: The future of the European Court of Human Rights: proceedings tof thet High-level Conference organised in Izmir, Turkey, on 26 and 27 April 2011 by the Turkish chairmanship of the Committee of Ministers of the Council of Europe, Council of Europe, Strasbourg, 2014.

- COMMitTEe of Ministers: The right to trial within a reasonable time and short-term reform of the European Court of Human Rights: round table, Bled, Slovenia, 21-22 September 2009, organised by the Slovenian Chairmanship of the Committee of Ministers of the Council of Europe, Ministry of Justice of the Republic of Slovenia: Ministry of Foreign Affairs of the Republic of Slovenia, Ljubljana, 2009.

Cucarella Galiana, L.A.: «El proceso de amparo ante el Tribunal Europeo de Derechos Humanos (1)», Diario La Ley, n. ${ }^{\circ}$ 8349, 2014.

DE VyLDER, H.: «The duty of cooperation of the respondent state during the proceedings before the European Court of Human Rights», HAECK, Y. and Brems, E. (eds.): Human rights and civil liberties in the 21st century, Springer, Dordrecht; Heidelberg; New York, 2014, pp. 37-67.

De Boer-Buquicchio, M.: «Procedures and administration in the Registry of the European Court of Human Rights», The Council of Europe's 50th anniversary: legal seminar, Oslo 27 May 1999, University of Oslo, Faculty of Law- Norwegian Institute of Human Rights, Oslo, 2000, pp. 25-35.

Díaz Barrado, C.M.: «Las reformas procedimentales: el Tribunal Europeo de Derechos Humanos», Bustamante RuA, M.M. (coord.): La jurisdicción y la protección internacional de los derechos, Universidad de Medellín, 2011, pp. 169-183.

DonALD, A.: Responding to systemic human rights violations: an analysis of «pilot judgments» of the European Court of Human Rights and their impact at national level: report of Seminar at the European Court of Human Rights, Strasbourg, 14 June 2010, London Metropolitan University, London, 2010.

European Court of Human Rights. Department of the Jurisconsult: Bringing a case to the European Court of Human Rights: a practical guide on admissibility criteria, 3rd ed., Wolf Legal Publishers (WLP), Strasbourg, 2014.

Forowicz, M. (et alii) (eds.): La défense des requérants devant la Cour européenne des droits de l'bomme, Anthemis, Limal; Nemesis, Bruxelles, 2012.

2 Versiones en inglés y en francés. 
Fribergh, E.: «The future of the Court after Interlaken», Spielmann, D.; Tsirli, M. and Voyatzis, P. (eds.): La Convention européenne des droits de l'bomme, un instrument vivant: mélanges en l'honneur de Christos L. Rozakis I = The European Convention on Human Rights, a living instrument: essays in honour of Christos L. Rozakis, Bruylant, Bruxelles, 2011, pp. 199-206.

Fraser, J.: «Conclusion: the European Convention on Human Rights as a common European endeavor», Flogaitis, S.; ZwarT, T. and Fraser, J.: The European Court of Human Rights and its discontents: turning criticism into strength, Edward Elgar, Cheltenham, 2013, pp. 192-210.

FYRNYS, M.: «Expanding competences by judicial lawmaking: the pilot judgment procedure of the European Court of Human Rights», Von Bogdandy, A. and VenzKe, I. (eds.): International judicial lawmaking: on public authority and democratic legitimation in global governance, Springer, Heidelberg, 2012, pp. 329-363.

Gay Montalvo, E.: «El recurso de amparo a partir de la Ley Orgánica del Tribunal Constitucional (LOTC) 6/2007: el Tribunal Constitucional y el Tribunal Europeo de Derechos Humanos», López Guerra, L. (et alii) (Coords.): El Tribunal Europeo de Derechos Humanos: una visión desde dentro: en homenaje al Juez Josep Casadevall, Tirant lo Blanch, Valencia 2015, pp. 177-189.

Gerards, J. and Terlouw, A.: «Solutions for the European Court of Human Rights: the amicus curiae project», Flogaitis, S.; ZWART, T. and Fraser, J: The European Court of Human Rights and its discontents: turning criticism into strength, Edward Elgar, Cheltenham 2013, pp. 158-182.

HaIDER, D.: The pilot-judgment procedure of the European Court of Human Rights, Martinus Nijhoff, Leiden; Boston, 2013.

Harmsen, R: «The reform of the Convention system: institutional restructuring and the (geo-) politics of human rights», Christofferesen, J. and Rask Madsen, M. (eds): The European Court of Human Rights between law and politics, Oxford University Press, Oxford; New York, 2011, pp.119-143.

HENDY, J.: «Procedure in the European Court of Human Rights (with a particular focus on cases concerning trade union rights)», Dorssemont, F.; LörCHER, K. and SCHÖMANN, I. (eds.): The European Convention on Human Rights and the employment relation, Hart, Oxford; Portland, 2013, pp. 61-91.

KUČERA, M.: «The role, status, duties, responsabilities and privileges of lawyers before the European Court of Human Rights», Coussirat-Coustère, V. (et alii): Réciprocité et universalité: sources et régimes du droit international des droits de l'homme: Mélanges en l'bonneur du Professeur Emmanuel Decaux, Pedone, Paris, 2017, pp. 749-759.

LEACH, P.: Taking a case to the European Court of Human Rights, 4th ed., Oxford University Press, Oxford, 2017.

López GuERrA, L.M: «Le protocole n. ${ }^{\circ} 16$ - une vraie-fausse solution?: un début de réponse», Burgorgue-Larsen, L. (dir.): Les défis de l'interprétation et de l'application des droits de l'homme: de l'owverture au dialogue, Pedone, Paris, 2017, pp. 365-370.

LÖRCHER, K.: «The future of the European Court of Human Rights in the light of the Brighton Declaration», Dorssemont, F., Lörcher, K. and Schömann, I. (eds.): The European Convention on Human Rights and the employment relation, Hart, Oxford; Portland, 2013, pp. 93-104.

MAHONEY, P.: «The European Court of Human Rights and its ever-growing caseload: preserving the mission of the Court while ensuring the viability of the individual petition system», Flogaitis, S.; Zwart, T. and Fraser, J (eds.): The European Court of Human Rights and its discontents: turning criticism into strength, Edward Elgar, Cheltenham, 2013, pp. 18-26.

Statement on case-overload at the European Court of Human Rights, European Law Institute (ELI), Vienna, 2012.

Martín-Retortillo Baquer, L: «El concepto «víctima de una violación de los derechos» como determinante para el acceso al Tribunal Europeo de Derechos Humanos», Homenaje al profesor José Antonio Escudero, Editorial Complutense, Vol. 1, 2012, pp. 741-772. 
Mengual I Mallol, A. M.: «El nou article 47 del Reglament del Tribunal i el procediment de jutge únic: filtres legítims o obstacles desmesurats», López Guerra, L. (et alii), (Coords.): El Tribunal Europeo de Derechos Humanos: una visión desde dentro: en homenaje al Juez Josep Casadevall, Tirant lo Blanch, Valencia, 2015, pp. 167-176.

Montesinos Padilla, C.: «La evolución del TEDH: ¿hacia dónde se dirige el modelo convencional de tutela de los Derecho Humanos?», Queralt JimÉneZ, A. (coord.): El Tribunal de Estrasburgo en el espacio judicial europeo, Thomas Reuters Aranzadi, Cizur Menor, 2013, pp. 51-84.

Moreno Fernández, J.I.: «Los recursos ante el Tribunal Europeo de Derechos Humanos», Chico De la Cámara, P. y Galán Ruiz, J. (dirs.): La revisión de actos en materia tributaria, Lex Nova, 2016, pp. 965-992.

Morte Gómez, C.: Cómo presentar una demanda ante el Tribunal Europeo de Derechos Humanos: condiciones de admisibilidad y modificaciones recientes del procedimiento $2 \mathrm{a}$ ed. Tirant lo Blanch, Valencia, 2014.

—El procedimiento ante el Tribunal Europeo de Derechos Humanos», García Becedas, G. y Nogueira Guastavino, M. (dirs.): Lecciones de Jurisdicción Social, 2. ${ }^{a}$ ed., Tirant Lo Blanch, 2016, pp. 903-932.

NaIsmith, S.: «Exhaustion of domestic remedies: must the remedy be capable of directly addressing the Convention complaint?», López Guerra, L. (et alii), (Coords.): El Tribunal Europeo de Derechos Humanos: una visión desde dentro: en homenaje al Juez Josep Casadevall, Tirant lo Blanch, Valencia, 2015, pp. 157-166.

O'BOyLe, M.: What cases should the ECHR deal with? Filtering mechanism and admissibilty criteria: Oslo meeting, 7 December 2009, Council of Europe, Strasbourg, 2009.

O'meara, N.: «Reforming the European Court of Human Rights: the impacts of Protocols 15 and 16 to the ECHR», Ziegler, K. S.; Wicks, E. and Hodson, L. (eds.): The UK and European human rights: a strained relationship?, Hart, Oxford; Portland, 2015, pp. 71-94.

QuAST MERTSCH, A.: «The reform measures of ECHR Protocol No. 14 and the provisional application of treaties», Fitzmaurice, M. and Merkouris, P. (eds.): The interpretation and application of the European Convention of Human Rights: legal and practical implications, Nijhoff, M., Leiden; Boston, 2013, p. 33.

Quesada, S.: «2010-2015: cinco años de aplicación del Protocolo n. ${ }^{\circ}$ 14: del Protocolo n. ${ }^{\circ} 11$ (1998) al Protocolo n. ${ }^{\circ} 14$ (2010)», RAImONDI, G. (et alii) (Eds.): Human rights in a global world: essays in honour of judge Luis López Guerra L., Wolf Legal Publishers, Oisterwijk, 2018, pp. 89-103.

—Las obligaciones del abogado ante el TEDH», López Guerra, L. (et alii) (Coords): El Tribunal Europeo de Derechos Humanos: una visión desde dentro: en homenaje al Juez Josep Casadevall, Tirant lo Blanch, Valencia 2015, pp. 137-156.

Ruiloba Alvariño, J.: «El Tribunal europeo de derechos humanos: aspectos organizativos y funcionales», Sempre Navarro, A.V. (dir.); Meléndez Morillo-Velarde, L. (coord.): Prontuario de jurisprudencia social del Tribunal europeo de derechos humanos (1975-2009), Aranzadi, Cizur Menor, 2009, pp. 103-189.

Salinas Alcega, S.: El sistema europeo de protección de los derechos bumanos en el siglo XXI: el proceso de reforma para asegurar su eficacia a largo plazo, Iustel, Madrid, 2009.

SPINOSI, P.: «La transparence de la pratique interne de la Cour européenne des droits de l'homme», Touzé, S. (dir.): La Cour européenne des droits de l'homme: une confiance nécessaire pour une autorité renforcée: actes du colloque des 19-20 novembre 2015, Pedone, Paris, 2016, pp. 167-178.

STOLFI, F.: Future developments of the European Court of Human Rights in the light of the Wise Persons' Report: proceedings tof the) colloquy, San Marino, 22-23 March 2007, Council of Europe, Strasbourg, 2007.

Torres PÉrez, A.: «El pronunciamiento previo del TJUE ¿un diálogo forzado?», Queralt JimÉnEZ, A. (coord.): El Tribunal de Estrasburgo en el espacio judicial europeo, Thomas Reuters Aranzadi, Cizur Menor, 2013, pp. 85-108. 
Thorarensen, B.: «The advisory jurisdiction of the ECtHR under protocol No.16: enhancing domestic implementation of human rights or a symbolic step?», MJöll ARNARDótTir, O. and BUYSE, A. (eds.): Shifting centres of gravity in human rights protection: retbinking relations between the ECHR, EU and national legal orders, Routledge, Abingdon; Oxon; New York, 2016, pp. 79-100.

WILDHABER, L.: «Pilot judgments in cases of structural or systematic problems on the national level», Wolfrum, R., and Deutsch, U. (eds.): The European Court of Human Rights overwhelmed by applications: problems and possible solutions, Springer, Berlin; Heidelberg; New York, 2009, pp. 69-92.

\section{Artículos de revistas}

Abrisketa Uriarte, J.: «Las sentencias piloto: el Tribunal Europeo de Derechos Humanos, de juez a legislador», Revista española de derecho internacional, Vol. 65, n. ${ }^{\circ}$ 1, 2013, pp. 73-99.

BAĞLAYAN, B. and HENDRIK FAHNER, J.: « «One can always do better»: the referral procedure before the Grand Chamber of the European Court of Human Rights», Human rights law review, vol. 17, no. 2, 2017, pp. 339-363.

BAyONA I Rocamora, A.: «La impugnación de la Sentencia: posibilidades de acceso al Tribunal Europeo de Derechos Humanos», Revista catalana de dret públic, n. ${ }^{\circ}$ Extra 1, 2010, pp. $475-478$.

Bolard, G.: «La Cour européenne des droits de l'homme et les formes de procédures», Recueil Dalloz, 192e année, n. $^{\circ} 21$, juin 2016, pp. 1245-1247.

BouazZa AriÑo, O.: «Condiciones de admisibilidad de las demandas presentadas ante el Tribunal Europeo de Derechos Humanos», Revista General de Derecho Administrativo, n. ${ }^{\circ}$ 29, 2012.

Cacho SÁnchez, Y.: «Cómo mejorar la eficacia del Tribunal Europeo de Derechos Humanos: las medidas propuestas por el Grupo de Sabios en su Informe de 15 de noviembre de 2006», Revista General de Derecho Europeo, n. ${ }^{\circ}$ 12, 2007.

CAFlisCH, L.: «La Cour européenne des droits de l'homme: un chantier permanent?», Zeitschrift für schweizerisches Recht $(Z S R)=$ Revue de droit suisse $(R D S)=$ Rivista di diritto svizzero $=$ Revista da dregt svizzer, Bd. 131, [Halbbd.] 1, H. 2, 2012, pp. 159-183.

Cano Palomares, G.: «La existencia de un perjuicio importante como nueva condición de admisibilidad tras la entrada en vigor del protocolo num. 14 al CEDH», Revista española de derecho Europeo, nr. 42, abril-junio 2012, pp. 49-73.

Costa, J.P.: «Les réformes de la Cour européenne des droits de l'homme: avant et après Brighton», L'observateur de Bruxelles, n. ${ }^{\circ} 89,2012$, pp. 29-34.

Crépet Daigremont, C.: «Nouvelle étape de la réforme de la Cour européenne des droits de l'homme: vers une redistribution des rôles de la Cour et des Etats», Annuaire français de droit international, vol. 58, 2012, pp. 655-677.

Chueca Sancho, A.G.: «El nuevo Tribunal Europeo de Derechos Humanos: la entrada en vigor del Protocolo Adicional núm. 14 en junio de 2010», Revista de derecho migratorio y extranjería, n. ${ }^{\circ} 24,2010$, pp. 217-230.

De LoNDRAs, F.: «Dual functionality and the persistent frailty of the European Court of Human Rights», European human rights law review, issue 1, 2013, pp. 38-46.

Dothan, S.: «Judicial tactics in the European Court of Human Rights», Chicago journal of international law, vol. 12, no. 1, summer 2011, pp. 115-142.

Dourneau-JosetTe, P.: «Le protocole 14 à la CEDH amendant le système de contrôle de la Convention», Actualité juridique pénal, n. ${ }^{\circ}$ 7-8, juil.-août 2004, pp. 283-284.

GLAS, L.R.: «Changes in the procedural practice of the European Court of Human Rights: consequences for the Convention system and lessons to be drawn», Human rights law review, vol. 14, nr. 4, 2014, pp. 671-699. 
GonZalez, G.: «Rêveries aquatiques sur le devenir de la Cour européenne des droits de l'homme», La Semaine juridique: édition générale (JCP), 86e année, nr. 19, mai 2012, pp. 943-945.

GRAGL, P.: «(Judicial) love is not a one-way street: the EU preliminary reference procedure as a model for ECtHR advisory opinions under draft Protocol no. 16», European law review, vol. 38, nr. 2, 2013, pp. 229-247.

Gerards, J.: «Advisory opinions, preliminary rulings and the new Protocol no. 16 to the European Convention of Human Rights: a comparative and critical appraisal», Maastricht journal of European and comparative law, vol. 21, no. 4, 2014, pp. 630-651.

Greer, S., Wylde, F.: «Has the European Court of Human Rights become a «small claims tribunal» and why, if at all, does it matter?», European buman rights law review, issue 2, 2017, pp. $145-154$.

Haeck, Y., Burbano Herrera, C. and ZwaAk, L.: «Non-compliance with a provisional measure automatically leads to a violation of the right of individual application... or doesn't it?: Strasbourg Court takes away any remaining doubts and broadens its pan-European protection», European Constitutional Law Review, vol.4, issue 1, 2008, pp. 41-63.

HAECK, Y.: «Desperately trying to keep the Titanic afloat: the reform proposals concerning the European Convention on Human Rights after Protocol No. 14: the report of the group of wise persons - and some further proposals», Inter-American and European buman rights journal = Revista interamericana y europea de derechos humanos, vol. 1, nr. 1, 2008, pp. 87-123.

JACQUÉ, J.P.: «Les Cours européennes sur la voie de la réforme?», Revue trimestrielle de droit européen, 48e année, n. ${ }^{\circ}$ 2, avril-juin 2012, pp. 289-293.

KRENC, F.: «"Dire le droit", "rendre la justice”: quelle Cour européenne des droits de l'homme?», Revue trimestrielle des droits de l'homme, 29e année, n. ${ }^{\circ} 114$, avril 2018, pp. 311-346.

—Quelques notes dubitatives sur le Protocole n. ${ }^{\circ} 16$ à la Convention européenne des droits de l'homme: pour un dépassement du «dialogue des juges», Annuaire international des droits de l'homme, vol. 9, 2015-2016, pp. 411-423.

Kurban, D.: «Forsaking individual justice: the implications of the European Court of Human Rights' pilot judgment procedure for victims of gross and systematic violations», Human rights law review, vol. 16, no. 4, 2016, pp. 731-769.

LAmbert AbDelgawad, E.: «The economic crisis and the evolution of the system based on the ECHR: is there any correlation?», European law journal, vol. 22, nr. 1, 2016, pp. 74-91.

- «Measuring the judicial performance of the European Court of Human Rights», International journal for court administration, vol. 8, nr. 2, May 2017, pp. 20-29.

—El Tribunal Europeo de Derechos Humanos y la técnica de las sentencias piloto: una pequeña revolución en marcha en Estrasburgo», Revista de Derecho Político, n. ${ }^{\circ}$ 69, 2007, pp. 355-383.

Larrouturou, T: «Le Protocole n. ${ }^{\circ} 16$ à la CEDH, nouveau terrain de rencontre des contrôles de constitutionnalité et de conventionnalité», Revue du droit public et de la science politique en France et à l'Etranger, vol. 134, n. ${ }^{\circ}$ 2, mars-avril 2018, pp. 475-497.

LEACH, P.: «On reform of the European Court of Human Rights», European buman rights law review, issue 6, 2009, pp.725-735.

López-Barajas Perea, I.: «Hacia una mayor eficacia del proceso de amparo internacional: el Protocolo n. ${ }^{\circ} 14$ a la Convención Europea de Derechos Humanos», Revista General de Derecho Europeo, n. ${ }^{\circ}$ 21, 2010.

López Guerra, L.M: «Los Protocolos de reforma n. ${ }^{\circ} 15$ y 16 al Convenio Europeo de Derechos Humanos», Civitas. Revista española de derecho europeo, n. ${ }^{\circ}$ 49, 2014, pp. 11-29.

LUCAS, K.: «Regard sur le processus de révision initié à Interlaken: entre (ré)action et inhibition», Revue trimestrielle des droits de l'bomme, 26e année, n. ${ }^{\circ} 101$, janv. 2015, pp. 65-88.

Martín-Retortillo BaQuer, L.: «El concepto «víctima de una violación de los derechos» como determinante para el acceso al Tribunal Europeo de Derechos Humanos», Revista de Administración Pública, n. ${ }^{\circ}$ 175, enero-abril, 2008, Madrid, pp. 253-284. 
Malinverni, G.: «Le Protocole n. ${ }^{\circ} 15$ à la Convention européenne des droits de l'homme», Revue trimestrielle des droits de l'homme, 26e année, n. ${ }^{\circ}$ 101, janv. 2015, pp. 51-63.

McCormick, P.: ««A risk of irreparable damage»: interim measures in proceedings before the European Court of Human Rights», Cambridge yearbook of European legal studies, vol. 12, 2009-2010, pp. 313-336.

Milner, D.: «Protocols no. 15 and 16 to the European Convention and Human Rights in the context of the perennial process of reform: a long and winding road», Zeitschrift für europarechtliche Studien (ZEUS), Jahrg. 17, H. 1, 2014, pp. 19-51.

Morawa, A.: «The European Court of Human Rights' rejection of petitions where the applicant has not suffered a significant disadvantage: a discussion of desirable and undesirable efforts to safeguard the operability of the Court», Journal of transnational legal issues, vol. 1, issue 1, Dec. 2012, pp. 1-19.

Morte Gómez, C.: «El Protocolo n. ${ }^{\circ} 14$ y la Conferencia de Interlaken: ¿Soluciones mágicas al colapso del TEDH?», Revista europea de derechos fundamentales, n. . 15, 2010, pp. 113-135.

Mowbray, A.: «The Interlaken Declaration: the beginning of a new era for the European Court of Human Rights?», Human rights law review, vol. 10, no. 3, 2010, pp. 519-528.

MÜLLER, L. F.: «The most recent development in the reform process of the control system of the European Convention on Human Rights: the Additional Protocol No. 14bis», Zeitschrift für ausländisches öffentliches Recht und Völkerrecht $(Z a \ddot{o} R V)=$ Heidelberg journal of international law (HJIL), Jahrg. 69, H. 2, 2009, pp. 397-402.

Netherlands Institute Of Human Rights (SIM): «High level conference on the future of the European Court of Human Rights: Brighton Declaration», Netherlands quarterly of buman rights, vol. 30, no. 3, Sept. 2012, pp. 349-362.

PASTOR RIDRUejo, J.A.: «La protección jurisdiccional de los derechos humanos: una comparación entre el tribunal europeo y la corte interamericana», REIB: Revista Electrónica Iberoamericana, Vol. 1, n. ${ }^{\circ}$ 1, 2007, pp. 5-13.

Pérez de los Cobos y OrIHuel, F.: «El recurso de amparo y el recurso ante el TEDH: pautas de interacción», Actualidad jurídica Uría Menéndez, n. ${ }^{\circ}$ 47, 2017, pp. 7-16.

O'Boyle, M. and Brady, N.: «Investigatory powers of the European Court of Human Rights», European human rights law review, issue 4, 2013, pp. 378-391.

Ortega Giménez, A., Pellicer Mollá, I. y Heredia Ortíz, P.: «¿Cómo se debe presentar una demanda ante el Tribunal Europeo de Derechos Humanos?», Economist E Jurist, Vol. 22, n. ${ }^{\circ} 178,2014$, pp. 22-30.

Paúl Díaz, A.: «Protocolo 14: Mejorando la eficiencia del tribunal europeo de derechos humanos», Revista chilena de derecho, Vol. 37, n. ${ }^{\circ}$ 3, 2010, pp. 613-615.

Queralt Jiménez, A.: «El Tribunal Europeo de Derechos Humanos: órgano y procedimiento (comunicación)», Estudios de derecho judicial, n. ${ }^{\circ}$ 155, 2009, pp.75-98.

RIETIKER, D.: «The European Court of Human Rights from a comparative law viewpoint: a logical example of a «mixed jurisdiction» Court», Cyprus human rights law review, vol. 2 , issue 1, 2013, pp. 36-52.

Rui, J. P.: «The Interlaken, Izmir and Brighton declarations: towards a paradigm shift in the Strasbourg Court's interpretation of the European Convention of Human Rights?», Nordic journal of buman rights, vol. 31, no. 1, 2013, pp. 28-54.

SÁrz ARNAIZ, A.: «De minimis non curat praetor: el «perjuicio importante» y la admisión de las demandas ante el Tribunal Europeo de Derechos Humanos», Revista Vasca de Administración Pública, n. ${ }^{\circ}$ 87-88, 2010, pp. 943-959.

SÁnChez PADrón, J.M.: «El recurso individual ante el Tribunal Europeo de Derechos Humanos: evolución y perspectiva», Revista europea de derechos fundamentales, $\mathrm{n} .{ }^{\circ} 18$, 2011, pp. 167-190.

SHELton, D.: «Significantly disadvantaged?: Shrinking access to the European Court of Human Rights», Human rights law review, vol. 16, no. 2, 2016, pp. 303-322. 
Tickell, A.: More «efficient» justice at the European Court of Human Rights: but at whose expense?, Public law, issue 2 (Apr. 2015), pp. 206-215.

—Dismantling the iron-cage: the discursive persistence and legal failure of a «bureaucratic rational» construction of the admissibility decision-making of the European Court of Human Rights» German law journal, vol. 12, no. 10, 2011, pp. 1786-181.

Tulkens, F.: «La Cour européenne des droits de l'homme et la Déclaration de Brighton: oublier la réforme et penser l'avenir», Cabiers de droit européen, n. ${ }^{\circ}$ 3, 2012, pp. 305-343.

«The link between manifest ill-foundedness and absence of a significant disadvantage as inadmissibility criteria for individual applications», The Italian yearbook of international law, vol. 20, 2010, pp. 169-179.

UZUn-MARINKović, A.: «Fostering domestication of human rights through the exhaustion of domestic remedies: a lesson learned from the ECtHR pilot and leading judgment procedures», Inter-American and European human rights journal = Revista interamericana y europea de derechos bumanos, vol. 9, no. 2, 2016, pp. 334-357.

VAN Den Eynde, L.: «An empirical look at the amicus curiae practice of human rights NGOs before the European Court of Human Rights», Netherlands quarterly of human rights, vol. 31, no. 3, Sept. 2013, pp. 271-313.

«The litigation practices of non-governmental organizations before the European Court of Human Rights: an overview», European yearbook on human rights, vol. 2011, pp. 539-548.

Wallace, S.: «Much ado about nothing?: The pilot judgment procedure at the European Court of Human Rights», European buman rights law review, issue 1, 2011, pp. 71-81.

\section{SENTENCIAS: CONTENIDO Y EFECTOS. EJECUCIÓN Y SUPERVISIÓN. MEDIDAS PROVISIONALES}

Libros y capítulos de libro

Arangüena Fanego, C.: «El cumplimiento de las sentencias del Tribunal Europeo de Derechos Humanos y la revisión de sentencias firmes», GARCía RoCA, F.J. y Fernández SÁNCHEZ, P.A. (coords.): Integración europea a través de derechos fundamentales: de un sistema binario a otro integrado, Centro de Estudios Políticos y Constitucionales, Madrid, 2009, pp. 289-325.

— «evisión penal y ejecución de las sentencias del tribunal europeo de derechos humanos», Arangüena Fanego, C. y SAnZ Morán, A.J. (coords.): La reforma de la justicia penal: aspectos materiales y procesales, Lex Nova, 2008, pp. 338-385.

Arangüena Fanego, C. y Landa Arroyo, C.: «Mecanismos procesales internos para la ejecución de las sentencias», García Roca, F.J., Carmona Cuenca, E. (coords.): ¿ Hacia una globalización de los derechos? el impacto de las sentencias del Tribunal Europeo y de la Corte Interamericana, Thomson Reuters-Aranzadi, 2017, pp. 169-209.

AкÇAY, D.: «The contribution of the government agent to the execution of judgments of the ECtHR [and] Discussion», Reforming the European Convention on Human Rights: a work in progress: a compilation of publications and documents relevant to the ongoing reform of the ECHR / prepared by the Steering Committee for Human Rights (CDDH), Council of Europe Publishing, Strasbourg, 2009, pp. 425-445.

Ayala Corao, C.M; Canosa Usera, R.L.: «El incumplimiento de las sentencias internacionales: problemas políticos y jurídicos», García Roca, F.J. y Carmona CuEnCA, E. (coords.): ¿Hacia una globalización de los derechos? el impacto de las sentencias del Tribunal Europeo y de la Corte Interamericana, Thomson Reuters-Aranzadi, 2017, pp. 269-312.

Cabrera Martín, M.: «Las medidas provisionales ante el Tribunal Europeo de Derechos Humanos: un análisis práctico con referencia a casos españoles», RAIMONDI, G. (ed.): Human 
rights in a global world: essays in honour of judge Luis López Guerra, Wolf Legal Publishers, Oisterwijk, 2018, pp. 251-265.

Council Of Europe. Parliamentary Assembly: The implementation of judgments of the European Court of Human Rights, Council of Europe Publishing, Strasbourg, 2018.

Council of Europe- Committee of Ministers: Annual Reports on the Supervision of the execution of judgments and decisions of the European Court of Human Rights. ${ }^{3}$

Cour Européenne Des Droits De L'homme: Dialogue entre juges: actes du séminaire 31 janvier 2014: la mise en oeuvre des arrêts de la Cour européenne des droits de l'bomme: une responsabilité judiciaire partagée?, Conseil de l'Europe, Strasbourg, 2014.

Casadevall, J.: «Medidas provisionales en el sistema europeo de protección de los derechos fundamentales», TOUZÉ, S. (dir.): L'abolition universelle de la peine de mort: actes du colloque des 9 et 11 octobre 2014, Pedone, Paris, 2016, pp.105-110.

De Albuquerque, P.P.: «Réflexions sur le renforcement de l'obligation d'exécution des arrêts de la Cour européenne des droits de l'homme», TOUZÉ, S. (dir.): La Cour européenne des droits de l'homme: une confiance nécessaire pour une autorité renforcée: actes du colloque des 19-20 novembre 2015, Pedone, Paris, 2016, pp. 217-226.

Donald, A. and Leach, P.: Parliaments and the European Court of Human Rights, Oxford University Press, Oxford, 2016.

Donald, A.: «The role of parliaments following judgments of the European Court of Human Rights», Hunt, M., Hooper, H.J. and Yowell, P. (eds.): Parliaments and human rights: redressing the democratic deficit, Hart, Oxford; Portland, 2015, pp. 59-92.

DrZEMCZEWSKI, A.: «Recent parliamentary initiatives to ensure compliance with Strasbourg Court judgments», LAMBERT-ABdelgawad, E. (at alii): L'bomme et le droit: en hommage au professeur Jean-François Flauss, Pedone, Paris, 2014, pp. 293-304.

EGaN, S.: «From judgment to compliance: domestic implementation of the judgments of the Strasbourg Court», Egan, S., Thornton, L. and Walsh, J.: Ireland and the European Convention on Human Rights: 60 years and beyond, Bloomsbury Professional, Haywards Heath 2014, pp. 17-36.

FERNÁNDEZ SÁNCHEZ, P.A.: «Las sentencias de interpretación: (Análisis comparado del Tribunal Europeo de Derechos Humanos y la Corte Interamericana de Derechos Humanos)», Hinojo Rojas, M. (coord.): Liber Amicorum profesor José Manuel Peláez Marón: Derecho Internacional y Derecho de la Unión Europea, Servicio de publicaciones de la Universidad de Córdoba, Cordoba, 2012, pp. 343-374.

FORTAS, A.C.: La surveillance de l'exécution des arrêts et décisions des cours européenne et interaméricaine des droits de l'homme: contribution à l'étude du droit du contentieux international, Pedone, Paris, 2015.

García Roca, J. y Nogueira Alcalá, H.: «El impacto de las sentencias europeas e interamericanas: valor de precedente e interpretación vinculante», GARCÍA ROCA, F.J., CARMONA CuencA, E. (coords.): ¿Hacia una globalización de los derechos? el impacto de las sentencias del Tribunal Europeo y de la Corte Interamericana, Thomson Reuters-Aranzadi, 2017, pp. 71-132.

GARLICKI, L.: «Judgments of the european court of human rights: (their structure, impact and authority)», García Roca, F.J., CARmona Cuenca, E. (coords.): ¿Hacia una globalización de los derechos? el impacto de las sentencias del Tribunal Europeo y de la Corte Interamericana, Thomson Reuters-Aranzadi, 2017, pp. 41-67.

GAVARA, J.C.: «L'intégration de la jurisprudence de la Cour européenne des droits de l'homme dans la jurisprudence constitutionnelle espagnole», SALES, M., y GAVARA, J.C. (et alii) (coords.): El sistema multinivel de los derechos fundamentales en Europa: 50 aniversario del Tribunal Europeo de Derechos Humanos, E-ditamos, Madrid, 2011.

3 Informes publicados anualmente en francés y en inglés. 
Gerards, J. (ed.): Implementation of the European Convention on Human Rights and of the judgments of the ECtHR in national case law: a comparative analysis, Intersentia, Cambridge; Antwerp; Portland, 2014.

GIL GIL, A.: «Eficacia de las decisiones del Tribunal Europeo de Derechos Humanos en el ámbito penal y procesal penal en España», Elsner, G; AMBOs, K. y MALARINo, E. (coords): Sistema interamericano de protección de los derechos humanos y derecho penal internacional, Vol. 3, Fundación Konrad-Adenauer, 2013, pp. 277-302.

GonZÁlez Alonso, A.: «La transformación de los ordenamientos procesales como consecuencia de la doctrina del Tribunal Europeo de Derechos Humanos», ReviRIEgo Picón, F. (coord.): Constitución y globalización: transformaciones del estado constitucional, Fundación Manuel Giménez Abad de Estudios Parlamentarios y del Estado Autonómico, 2013, pp. 143-163.

HEIKKILÄ, S.: From final judgment to final resolution: effectiveness of the execution of judgments of the European Court of Human Rights in Finland, Wolf Legal Publishers (WLP), Oisterwijk, 2016.

ICHIM, O.: Just satisfaction under the European Convention on Human Rights, Cambridge University Press, Cambridge, 2015.

Irurzun Montoro, F.: «La ejecución de las sentencias del Tribunal Europeo de Derechos Humanos: una aproximación a la práctica española», Queralt Jiménez, A. (coord.): El Tribunal de Estrasburgo en el espacio judicial europeo, Thomas Reuters Aranzadi, Cizur Menor, 2013 , pp. 131-162.

Keller, H. and STONe SweET, A. (ed.): A Europe of rights: the impact of the ECHR on national legal systems, Oxford University Press, Oxford; New York, 2008.

KRENC, F. (ed.): Les mesures provisoires devant la Cour européenne des droits de l'homme: un référé à Strasbourg?: \{Colloque, 18 mars 2011\}, Larcier, Bruxelles, 2011.

Lambert Abdelgawad, E.: The execution of judgments of the European Court of Human Rights 2 nd ed., Council of Europe, Strasbourg, 2008.

LEACH, P. (et alii): Responding to systemic human rights violations: an analysis of «pilot judgments» of the European Court of Human Rights and their impact at national level, Intersentia, Antwerp; Oxford; Portland, 2010.

L'institut International Des Droits De L'homme: Les mutations de l'activité du Comité des Ministres: la surveillance de l'exécution des arrêts de la Cour européenne des droits de l'bomme par cet organe du Conseil de l'Europe: actes du séminaire de l'Institut international des droits de l'bomme René Cassin, Anthemis, Limal; Nemesis, Bruxelles, 2012.

Martín Queralt, J.B.: «El derecho tributario español y el Tribunal Europeo de Derechos Humanos de Estrasburgo», ORDóÑEz Solís, D. (coord.): La recepción del derecho de la Unión Europea en España: derechos, mercado único y armonización fiscal en Europa: LIBER AMICORUMм en bomenaje a Antonio Martínez Lafuente, La Ley, Madrid, 2013, pp. 93-113.

Milione Fugali, C.: ««Algo queda por hacer...» La ejecución de las sentencias del Tribunal Europeo de Derechos Humanos en el ordenamiento jurídico español», Gordillo PÉrez, L.I. (coord.): Constitutionalism of European Supranational Courts: recent developments and challenges, Thomson Reuters-Aranzadi, 2015, pp. 81-105.

Morte Gómez, C.: «Diez años de jurisprudencia del Tribunal Europeo de Derechos Humanos en casos españoles: temas tratados, evolución, efectos», RAIMONDI, G. (et alii) (eds): Human rights in a global world: essays in honour of judge Luis López Guerra, Wolf Legal Publishers, Oisterwijk 2018, pp. 281-301.

— «Eficacia de las sentencias del Tribunal Europeo de Derechos Humanos», Cursos de derechos humanos de Donostia-San Sebastián, Vol. 11, Universidad del País Vasco/Euskal Herriko Unibertsitatea, Servicio de Publicaciones, 2011, pp. 225-242.

Otazua Zabala, G.: «La recepción de la doctrina del Tribunal Europeo de Derechos Humanos por el Tribunal Constitucional: El principio de publicidad en los recursos penales», GoIzueta Vértiz, J. y Cienfuegos Mateo, M. (dirs.) La eficacia de los derechos fundamentales de la UE: cuestiones avanzadas, Thomson Reuters-Aranzadi, 2014, pp. 189-214. 
PÉrez Sola, N.: «El Tribunal constitucional como juez de los derechos y la integración de la jurisprudencia del Tribunal Europeo de Derechos Humanos: el estado de la cuestión», Pérez Tremps, P. (coord.): La Reforma del Tribunal Constitucional: actas del V Congreso de la Asociación de Constitucionalistas de España, Tirant lo Blanch, Valencia, 2007, pp. 793-822.

Queralt Jiménez, A.: La interpretación de los derechos: del Tribunal de Estrasburgo al tribunal constitucional, Centro de estudios políticos y constitucionales, Madrid, 2008.

Ripol Carulla, S.: España en Estrasburgo: tres décadas bajo la jurisdicción del Tribunal Europeo de Derechos Humanos, Aranzadi, Cizur Menor, 2010.

- El sistema europeo de protección de los derechos humanos y el derecho español: la incidencia de las sentencias del Tribunal Europeo de Derechos Humanos en el ordenamiento jurídico español, Atelier, Barcelona, 2007.

—La ejecución de las sentencias del Tribunal Europeo de Derechos Humanos en el ordenamiento jurídico español», De La Cuesta Arzamendi, J.L. y Pérez Machío, A.I. Ugartemendía Eceizabarrena, J.I (dirs.): Armonización penal en Europa, Gobierno Vasco, Instituto Vasco de Administración Pública = Herri Arduralaritzaren Euskal Erakundea, 2013, pp. 514-546.

Rodríguez Boente, S.E.: 83 argumentos que convencen al Tribunal Europeo de Derechos Humanos: jurisprudencia que afecta a España, Aranzadi, Cizur Menor, 2015.

Saavedra Alessandri, P., Cano Palomares, G. y Hernández Ramos, M.: «Reparación y supervisión de sentencias», García Roca, F.J., Carmona Cuenca, E. (coords.): ¿ Hacia una globalización de los derechos? el impacto de las sentencias del Tribunal Europeo y de la Corte Interamericana, Thomson Reuters-Aranzadi, 2017, pp. 211-268.

SaIz Arnaiz, A.: «El recurso de revisión penal y la ejecución de las sentencias condenatorias del Tribunal Europeo de Derechos Humanos», López Guerra, L. (et alii) (coords.): El Tribunal Europeo de Derechos Humanos: una visión desde dentro: en homenaje al Juez Josep Casadevall, Tirant lo Blanch, Valencia, 2015, pp. 199-212.

Salado Osuna, A.: «La ejecución de las sentencias indemnizatorias del Tribunal Europeo de Derechos Humanos y del Tribunal de Justicia de las Comunidades Europeas», García RoCA, F.J. y FERNÁNDEZ SÁNCHEZ, P.A. (coords.): Integración europea a través de derechos fundamentales: de un sistema binario a otro integrado, Centro de Estudios Políticos y Constitucionales, Madrid, 2009, pp. 257-288.

Seibert-Fohr, A. and Villiger, M.E. (eds.): Judgments of the European Court of Human Rights: effects and implementation, Ashgate, Baden-Baden: Nomos, 2014.

SobCZAK, J.: «Execution of judgments: problem of effectiveness monitoring of the execution of the ECHR judgments», Coulcil Of Europe-Steering Committee For Human Rights (CDDH): Reforming the European Convention on Human Rights: a work in progress: a compilation of publications and documents relevant to the ongoing reform of the ECHR, Council of Europe Publishing, Strasbourg 2009, pp. 183-187.

SUNDBERG, F.: «Effective remedies, notably in the case of unreasonable length of proceedings: recent developments in the Committee of Ministers' supervision of the execution of judgments», Coulcil Of Europe-Steering Committee For Human Rights (CDDH): Reforming the European Convention on Human Rights: a work in progress: a compilation of publications and documents relevant to the ongoing reform of the ECHR, Council of Europe Publishing, Strasbourg, 2009, pp. 99-100.

- -Control of execution of decisions under the European Convention on Human Rights: a perspective on democratic security, inter-governmental cooperation, unification and individual justice in Europe», Alfredsson, G. (et alii)(eds.): International human rights monitoring mechanisms: essays in honour of Jakob Th. Möller, Martinus Nijhoff Publishers, Leiden; Boston, 2009, pp. 465-485.

SzYMCZAK, D.: «La question de l'acceptabilité des arrêts de la Cour européenne des droits de l'homme par les Etats parties», Touzé, S. (dir.): La Cour européenne des droits de l'bomme: une 
confiance nécessaire pour une autorité renforcée: actes du colloque des 19-20 novembre 2015, Pedone, Paris, 2016, pp. 123-139.

Touzé, S.: «Regard critique sur l'exécution conditionnelle des arrêts de la Cour européenne des droits de l'homme», Coussirat-Coustère, V. (dir.): Réciprocité et universalité: sources et régimes du droit international des droits de l'homme: Mélanges en l'honneur du Professeur Emmanuel Decaux, Pedone, Paris, 2017, pp. 761-777.

ZANGHì, C.: «Evolución e innovación de los efectos de las sentencias del Tribunal Europeo de los Derechos Humanos», García Roca, F.J. y Fernández Sánchez, P.A. (coords.): Integración europea a través de derechos fundamentales: de un sistema binario a otro integrado, Centro de Estudios Políticos y Constitucionales, Madrid, 2009, pp.199-228.

Ziemele, I.: Separate opinions at the European Court of Human rights, Riga Graduate School of Law, Riga, 2017.

Artículos

Blay-GrabarczyK, K.: «Un nouveau bilan encourageant dans le domaine de l'exécution des arrêts de la Cour EDH: à propos du 11e rapport annuel du Comité des ministres du Conseil de l'Europe», La Semaine juridique: édition générale (JCP), 92e année, n. ${ }^{\circ}$ 22, mai 2018, pp. 1043-1046.

«Le bilan intermédiaire des résultats obtenus dans le domaine de l'exécution des arrêts de la CEDH: à propos du 10e rapport annuel du Comité des ministres du Conseil de l'Europe», La Semaine juridique: édition générale (JCP), 91e année, n. ${ }^{\circ}$ 2, mai 2017, pp. 961-963.

BouazZa AriÑo, O.: «El derecho del Consejo de Europa en la europeización del derecho público español», Revista de administración pública, n. ${ }^{\circ}$ 204, 2017, pp. 103-131.

Burbano Herrera, C.: «Análisis Crítico de los Trabajos de la Comisión Europea y de la Corte Europea de Derechos Humanos en relación con el incumplimiento de las medidas provisionales - Periodo 1957-2011», Via Inveniendi et Iudicandi, Vol. 7, n. ${ }^{\circ}$ 2, 2012, pp. 190-246.

BYCHAWSKA-SINIARSKA, D.: "Why (and how) the Committee of Ministers needs to be reformed in order to enhance implementation of ECtHR judgments», European yearbook on human rights, vol. 2013, pp. 313-321.

CAFLISCH, L.: «New practice regarding the implementation of the judgments of the Strasbourg Court», The Italian yearbook of international law, vol. 15, 2005, pp. 3-23.

Cruz Ortiz De Landézuri, L.M.: «La reparación a las víctimas en el Convenio Europeo de Derechos Humanos», Revista española de derecho internacional, Vol. 62, n. ${ }^{\circ}$ 1, 2010, pp. 89-117.

De Londras, F.: «Mission impossible?: Addressing non-execution through infringement proceedings in the European Court of Human Rights», International and comparative law quarterly, vol. 66, pt. 2, Apr. 2017, pp. 467-490.

De Miguel Canuto, E.: «Eficacia interna de las sentencias del Tribunal Europeo de Derechos Humanos», Quincena fiscal, n. ${ }^{\circ} 19,2013$, pp. 61-83.

DrZemCZEwsKi, A.: «The Parliamentary Assembly's involvement in the supervision of the judgments of the Strasbourg Court», Netherlands quarterly of buman rights, vol. 28, no. 2, June 2010, pp. 164-178.

FossAs, E.: « «Cosa interpretada» en derechos fundamentales: jurisprudencia del TEDH y jurisprudencia constitucional», Revista Vasca de Administración Pública. Herri-Arduralaritzako Euskal Aldizkaria, n. ${ }^{\circ} 82,2$, 2008, pp. 165-180.

Garberí Llobregat, J.: «La ejecución en España de las sentencias del Tribunal Europeo de Derechos Humanos (1)», Diario La Ley, n. ${ }^{\circ}$ 8178, 2013.

García San Martín, J.: «La ejecución penal en España de las Sentencias del Tribunal Europeo de Derechos Humanos», Revista General de Derecho Procesal, n. ${ }^{\circ}$ 36, 2015. 
García Murcia, J.: «Virtualidad en el ordenamiento laboral de la jurisprudencia del Tribunal Europeo de Derechos Humanos (1)», Actualidad laboral, n. ${ }^{\circ}$ 6, 2014, p. 2.

Helander Capalbo, N.: «Efectos de la sentencias de la Corte Interamericana y del Tribunal Europeo de Derechos Humanos, con especial referencia a Uruguay y España», Revista IIDH, no. 55, enero-jun. 2012, pp. 207-231.

Hess, B.: «La influencia del Tribunal Europeo de Derechos Humanos en el Derecho procesal civil europeo», Anuario español de derecho internacional privado, n. ${ }^{\circ}$ 14-15, 2014-2015, pp. 35-53.

JAHN, J.: «Ruling (in)directly through individual measures? Effect and legitimacy of the ECtHR's new remedial power?», Zeitschrift für ausländisches öffentliches Recht und Völkerrecht (Zä̈RV) = Heidelberg journal of international law (HJIL), Jahrg. 74, H. 1, 2014, pp. 1-39.

Kurban, D.: «Forsaking individual justice: the implications of the European Court of Human Rights' pilot judgment procedure for victims of gross and systematic violations», Human rights law review, vol. 16, no. 4, 2016, pp. 731-769.

LAMBERT-ABDELGAWAD, E.: «Taking the implementation of ECtHR judgments seriously: right assessment, wrong approaches?», EHRAC Bulletin, issue 14, winter 2010, pp. 14-15.

- «The execution of the judgments of the European Court of Human Rights: towards a non-coercive and participatory model of accountability», Zeitschrift für ausländisches öffentliches Recht und Völkerrecht (Zä̈RV) = Heidelberg journal of international law (HJIL), Jahrg. 69, H. 3, 2009, pp. 471-506.

—L'exécution des arrêts de la Cour européenne des Droits de l'Homme», Revue trimestrielle des droits de l'homme, 18e année, n. ${ }^{\circ} 71$, juil. 2007, pp. 669-705.

LOUCAIDES, L.G.: «Reparation for violations of human rights under the European Convention and restitutio in integrum», European buman rights law review, issue 2, 2008, pp. 182-192.

Méndez Tojo, R.: «La ejecución en España de las sentencias del Tribunal Europeo de Derechos Humanos: ¿una reforma legal necesaria?», Diario La Ley, n. ${ }^{\circ}$ 8639, 2015.

Miara, L.: «The role of civil society in the execution of judgments of the European Court of Human Rights», European buman rights law review, issue 5, 2012, pp. 528-537.

Moliner Vicente, C.: «Las sentencias del Tribunal Europeo de Derechos Humanos en relación con España en 2013», Revista jurídica Universidad Autónoma de Madrid, n. ${ }^{\circ} 30$, 2014, pp. 305-330.

Montesinos Padilla, C.: «El recurso de revisión como cauce de ejecución de las sentencias del Tribunal de Estrasburgo: pasado, presente y futuro», Eunomía: Revista en Cultura de la Legalidad, n. ${ }^{\circ} 10,2016$, pp. 98-113.

Mowbray, A.: «An examination of the European Court of Human Rights' indication of remedial measures», Human rights law review, vol. 17, no. 3, 2017, pp. 451-478.

Paraskeva, C.: «The developing role of the European Court of Human Rights in the execution of its judgments: from declaratory judgments to indication of specific measures?», Annuaire international des droits de l'homme, vol. 9 (2015-2016), 2016, pp. 425-448.

«European Court of Human Rights: from declaratory judgments to indications of specific measures», European human rights law review, issue 1, 2018, pp. 46-56.

Quesada Alcalá, C.: «Las víctimas encuentran su lugar ante el Tribunal Europeo de Derechos Humanos: una «satisfacción equitativa» por la vulneración de sus derechos en las reclamaciones entre Estados (Chipre contra Turquía)», Revista de Derecho Comunitario Europeo, n. 49, septiembre/diciembre, 2014, pp. 889-915.

Ripol Carulla, S.: «Incidencia en la jurisprudencia del Tribunal Constitucional de las Sentencias del Tribunal Europeo de Derechos Humanos que declaran la vulneración por España del CEDH», Revista Española de Derecho Constitucional, n. ${ }^{\circ}$ 79, enero-abril, 2007, pp. 309-346. - «a ejecución de las Sentencias del Tribunal Europeo de Derechos Humanos en el ordenamiento jurídico español», Revista europea de derechos fundamentales, n. ${ }^{\circ}$ 15, 2010, pp. 75-112.

Rodríguez-PiÑero y Bravo-Ferrer, M.: «Un nuevo paso en la ejecución judicial de las sentencias del Tribunal Europeo de Derechos Humanos», Diario La Ley, n. . 8444, 2014. 
Ruiz Morales, M.L.: «El control de convencionalidad y los sistemas de protección de los derechos humanos americano y europeo. Su recepción en el caso argentino y español», Anuario iberoamericano de justicia constitucional, n. ${ }^{\circ} 21,2017$, pp. 129-160.

Simarro Pedreira, M.: «El nuevo motivo del recurso de revisión (especial referencia en el orden jurisdiccional penal)», Revista Jurídica de la Universidad de León, n. ${ }^{\circ}$ 3, 2016, pp. 27-54.

Salinas Alcega, S.: «El nuevo procedimiento de control de la ejecución de sentencias del Tribunal Europeo de Derechos Humanos tras el proceso de Interlaken: la evolución técnica de un mecanismo político», Revista General de Derecho Europeo, n. ${ }^{\circ}$ 36, mayo 2015.

SicilianOs, L.A.: «The involvement of the European Court of Human Rights in the implementation of its judgments: recent developments under Article 46 ECHR», Netherlands quarterly of human rights, vol. 32, no. 3, Sep. 2014, pp. 235-262.

Suchkova, M.: «An analysis of the institutional arrangements within the Council of Europe and within certain Member States for securing the enforcement of judgments», European buman rights law review, issue 4, 2011, pp. 448-463.

SzKLANNA, A.: «The impact of the pilot judgment procedure of the European Court of Human Rights on the execution of its judgments», European yearbook on buman rights, vol. 2010, pp. 223-232.

Tenorio SÁNCHEZ, P.J.: «Influence of the European Convention of Human Rights on the interpretation of the Spanish Constitution: an European perspective», Revista general de derecho público comparado, n. ${ }^{\circ}$ 7, 2010.

Torralba Mendiola, E.C.: «Sobre la eficacia en España de las resoluciones del Tribunal Europeo de Derechos Humanos», Derecho privado y Constitución, n. ${ }^{\circ} 21,2007$, pp. 313-330.

\section{PRINCIPIO DE SUBSIDIARIEDAD, MARGEN DE APRECIACIÓN NACIONAL Y CONSENSO EUROPEO}

Libros y capítulos de libros

AGHA, P. (ed.): Human rights between law and politics: the margin of appreciation in post-national contexts, Oxford; Portland, Hart, 2017.

Ask, B: Towards stronger implementation of the European Convention on Human Rights at national level: proceedings tof the) colloquy, Stockbolm, 9-10 June 2008: Council of Europe, Strasbourg, $2008^{4}$.

BESsON, S.: «L'évolution du contrôle européen: vers une subsidiarité toujours plus subsidiaire», Touzé, S. (dir.): La Cour européenne des droits de l'homme: une confiance nécessaire pour une autorité renforcée: actes du colloque des 19-20 novembre 2015, Pedone, Paris 2016, pp. 57-82.

ÇALI, B.: «From flexible to variable standards of judicial review: the responsible domestic courts doctrine at the European Court of Human Rights», Mjöll Arnardóttir, O. and BuYse, A. (eds.): Shifting centres of gravity in human rights protection: rethinking relations between the ECHR, EU and national legal orders, Routledge, Abingdon; Oxon; New York, 2016, pp. 161-188.

CosTA, J. P.: The role of government agents in ensuring effective human rights protection: proceedings (of the) seminar, Bratislava, 3-4 April 2008, Council of Europe, Strasbourg, 2008.

COUNCIL OF EUROPE: The role of government agents in ensuring effective human rights protection: proceedings (of the) seminar, Bratislava, 3-4 April 2008, Council of Europe, Strasbourg, 20085.

\footnotetext{
4 Versiones en inglés y en francés.

5 Versiones en inglés y en francés.
} 
CommitTeE Of Ministers: Implementation of the European Convention on Human Rights, our shared responsibility: proceedings tof the\} High Level Conference organised in Brussels, Belgium, 26-27 March 2015, Council of Europe, Strasbourg, $2015^{6}$.

Dzentsiarou, K.: European consensus and the legitimacy of the European Court of Human Rights, Cambridge University Press, Cambridge, 2015.

Dzentsiarou, K.: «Interaction between the European Court of Human Rights and member states: European consensus, advisory opinions and the question of legitimacy», FLogarTIS, S., ZWart T. and Fraser J. (eds.): The European Court of Human Rights and its discontents: turning criticism into strength, Edward Elgar, Cheltenham 2013, pp. 116-134.

Elens-Passos, F.: «Le consensus européen est-il tendance?», RAImONDI, G. (et alii) (eds.): Human rights in a global world: essays in honour of judge Luis López Guerra, Wolf Legal Publishers, Oisterwijk, 2018, pp. 5-19.

European Court Of Human Rights: Dialogue between judges: seminar 30 January 2015. Subsidiarity: a two-sided coin?, Council of Europe, Strasbourg, $2015^{7}$.

FABBRINI, F.: The margin of appreciation and the principle of subsidiarity: a comparison, University of Copenhagen, Centre of Excellence for International Courts (iCourts), Copenhagen, 2015.

Ferrer Mac Gregor, E. y Queralt Jiménez, A.: «El control de convencionalidad americano y el efecto de cosa interpretada europeo ¿dos caras de una misma moneda?», GARCÍA RocA, F.J. y Carmona Cuenca, E. (coords.): ¿Hacia una globalización de los derechos? el impacto de las sentencias del Tribunal Europeo y de la Corte Interamericana, Thomson Reuters-Aranzadi, 2017, pp. 133-168.

FolesDal, A.: «Squaring the circle at the battle at Brighton: is the war between protecting human rights or respecting sovereignty over, or has it just begun?», MJÖLL ARNARDótTIR O. and BUYSE A. (eds.): Shifting centres of gravity in human rights protection: rethinking relations between the ECHR, EU and national legal orders, Routledge, Abingdon; Oxon; New York, 2016, pp.189-204.

GARCía RocA, J.: «Criterios para la aplicación del margen de apreciación nacional en las sentencias del Tribunal Europeo de Derechos Humanos: el alcance del escrutinio en el amparo europeo», Inst. Iberoamericano D. ${ }^{\circ}$ Constitucional, Asociación Peruana D. ${ }^{\circ}$ ConStitucional, Instituto de Investigaciones Jurídicas, Maestría en Derecho ConSTITUCIOnal (coords).: Memoria X Congreso Iberoamericano de Derecho Constitucional: (Lima, 16-19 de septiembre de 2009), Vol. 1, Tomo 1, IDEMSA, 2009, pp. 737-762.

- El margen de apreciación nacional en la interpretación del Convenio Europeo de Derechos Humanos: soberanía e integración, Civitas-Thomson Reuters, Madrid, 2010.

— «a muy discrecional doctrina del margen de apreciación nacional según el Tribunal Europeo de Derechos Humanos: soberanía e integración», Aguilera Vaqués, M.: Estudios sobre la Constitución Española: homenaje al profesor Jordi Solé Tura, Vol. 2, Congreso de los Diputados, 2008, pp. 1951-1976.

«Soberanía estatal versus integración europea mediante unos derechos fundamentales comunes: ¿cuál es el margen de apreciación nacional?», GARCÍA RoCa F.J. y FernándeZ SÁNCHEZ, P.A. (coords.): Integración europea a través de derechos fundamentales: de un sistema binario a otro integrado, Madrid, 2009, Centro de Estudios Políticos y Constitucionales, pp. 15-53.

GonZalez G. (dir.): La subsidiarité conventionnelle en question: essai de systématisation, Anthemis, Limal; Nemesis, Bruxelles, 2016.

Hallström, P.: «Balance or clash of legal orders: some notes on margin of appreciation», Nergelius, J. and Kristoffersson, E.: Human rights in contemporary European law, Hart, Oxford; Portland, 2015, pp. 59-73.

6 Versiones en inglés y en francés.

7 Versiones en inglés y en francés. 
IMBERT, P. H.: «Follow-up to the Committee of Ministers' recommendations on the implementation of the Convention at the domestic level and the declaration on «Ensuring the effectiveness of the implementation of the ECHR at national and European level»», STEERING Committee For Human Rights (CDDH): Reforming the European Convention on Human Rights: a work in progress: a compilation of publications and documents relevant to the ongoing reform of the ECHR, Council of Europe Publishing, Strasbourg 2009, pp. 66-738.

McGolDRICK, D.: «Affording States a margin of appreciation: comparing the European Court of Human Rights and the Inter-American Court of Human Rights», BuCKley, C.M., Alice Donald, A. and LEACH, P. (eds.): Towards convergence in international buman rights law: approaches of regional and international systems, Brill Nijhoff, Boston, 2017, pp. 325-365.

Mjöll ARnardótTir, O. and GuĐmundsDótTir, D.: «Speaking the same language? Comparing judicial restraint at the ECtHR and the ECJ», MjöLl ArnardótTir, O. and Buyse, A. (eds.): Shifting centres of gravity in human rights protection: rethinking relations between the ECHR, $E U$ and national legal orders, Routledge, Abingdon; Oxon; New York, 2016, pp. 161-188.

Nussberger, A.: «Subsidiarity in the control of decisions based on proportionality: an analysis on the basis of the implementation of ECtHR judgments into German law», SEIBERT-FoHR A. and VILLIGER M.E. (eds.): Judgments of the European Court of Human Rights: effects and implementation, Nomos; Ashgate, Baden-Baden, 2014, pp. 165-185.

Petrović-ŠKero, V.: The role of Supreme Courts in the domestic implementation of the European Convention on Human Rights: proceedings (of the) regional conference, Belgrade, Serbia, 20-21 September 2007, Council of Europe, Strasbourg, $2008^{9}$.

Saiz Arnaiz, A.: «La protección de los derechos fundamentales en la jurisdicción ordinaria: una visión desde el Tribunal Europeo de Derechos Humanos», La protección jurisdiccional de los derechos. Actas del XI Congreso de la Asociación de Constitucionalistas de España, Tirant lo Blanch, Valencia, 2015, pp. 51-65.

Touzé, S. (ed): La Cour européenne des droits de l'homme une confiance nécessaire pour une autorité renforcée : actes du colloque des 19-20 novembre 2015, Pedone, Paris, 2016.

SpIElmanN, D.: «Le fait, le juge et la connaissance: aux confins de la compétence interprétative de la Cour européenne des droits de l'homme», D’Argent P. et Bonafé B., CombaCau J. (cords.): Les limites du droit international: essais en l'honneur de Joe Verboeven = The limits of international law: essays in honour of Joe Verhoeven, Bruylant, Bruxelles, 2015, pp. 519-535.

Sudre, F. (ed.): Le principe de subsidiarité au sens du droit de la Convention européenne des droits de l'homme: actes du colloque, Montpellier, 18-19 octobre 2013, Anthemis, Limal; Nemesis, Bruxelles, 2014.

VAN Hoof, F.: «The stubbornness of the European Court of Human Rights' margin of appreciation doctrine», HAECK, Y. (et alii) (eds.): The realisation of human rights: when theory meets practice: studies in honour of Leo Zwaak, Intersentia, Cambridge; Antwerp; Portland, 2013, pp. 125-149.

Walton, D.: «Subsidiarity and the Brighton Declaration», SeiberT-Fohr, A. and Villiger, M.E. (eds.): Judgments of the European Court of Human Rights: effects and implementation, Nomos; Ashgate, Baden-Baden, 2014, pp. 193-20.

Artículos de revistas

Alguacil GonZÁlez-Aurioles, J. A.: «Ponderación, proporcionalidad y margen de apreciación en la jurisdicción europea de los derechos», Revista General de Derecho Europeo, n. . 25, 2011.

8 Versiones en inglés y en francés.

9 Versiones en inglés y en francés. 
BATES, E.: «Activism and self-restraint: the margin of appreciation's Strasbourg career- its coming of age?», Human rights law journal (HRLJ), vol. 36, no. 7-12, 30 Dec. 2016, pp. 261-282.

Crépet Daigremont, C.: «Nouvelle étape de la réforme de la Cour européenne des droits de l'homme: vers une redistribution des rôles de la Cour et des Etats», Annuaire français de droit international, vol. 58, 2012, pp. 655-677.

De Montalvo JäÄskeläInen, F.: «El difícil equilibrio entre la globalización de los derechos y su contextualización desde la perspectiva de la doctrina del margen de apreciación nacional», Estudios de Deusto: revista de la Universidad de Deusto, Vol. 60, n. ${ }^{\circ}$ 2, 2012, pp.133-154.

Draghici, C.: «The Strasbourg Court between European and local consensus: anti-democratic or guardian of democratic process?», Public law, issue 1, Jan. 2017, pp. 11-29.

Giannopoulos, C.: «Subsidiarité procédurale et 16e Protocole additionnel à la Convention européenne des droits de l'homme», Annuaire international des droits de l'homme, vol. 9, 20152016, pp. 675-692.

HaRvey, P.: «The principle of subsidiarity: a more positive approach», Human rights law journal (HRLJ), vol. 36, no. 7-12, 30 Dec. 2016, pp. 335-339.

IgLEsias VILA, M.: «Subsidiariedad y tribunales internacionales de derechos humanos: ¿deferencia hacia los estados o división cooperativa del trabajo?», Derecho PUCP: Revista de la Facultad de Derecho, n. ${ }^{\circ}$ 79, 2017, pp. 191-222.

KuiJer, M.: «The margin-of-appreciation doctrine and the strengthening of the principle of subsidiarity in the recent reform negotiations», Human rights law journal (HRLJ), vol. 36, no. 7-12, 30 Dec. 2016, pp. 339-347.

LAmbrecht, S.: «Reforms to lessen the influence of the European Court of Human Rights», European public law, vol. 21, issue 2, June 2015, pp. 257-283.

Mowbray, A.: «Subsidiarity and the European Convention on Human Rights», Human rights law review, vol. 15, no. 2, 2015, pp. 313-341.

Mena Parras, F.J: «Democracy, diversity and the margin of appreciation: a theoretical analysis from the perspective of the international and constitutional functions of the European court of human rights», Revista electrónica de estudios internacionales (REEI), n. . 29, 2015.

NASH Rojas, C.: «La doctrina del margen de apreciación y su nula recepción en la jurisprudencia de la Corte Interamericana de Derechos Humanos», ACDI - Anuario Colombiano de Derecho Internacional, n. ${ }^{\circ} 11,2018$, pp. 71-100.

Pascual Vives, F. J.: «El margen de apreciación nacional en los tribunales regionales de derechos humanos: una aproximación consensualista $=$ The margin of appreciation in the case-law of the regional human rights courts: a consensualist approach», Anuario español de derecho internacional, vol. 29, 2013, pp. 217-262.

«Consenso e interpretación evolutiva de los tratados regionales de derechos humanos», Revista española de derecho internacional, Vol. 66, n. ${ }^{\circ}$ 2, 2014, pp. 113-153.

PASTRE-BELDA, B.: «Et si la Cour européenne des droits de l'homme renonçait à l'interprétation consensuelle?», Revue trimestrielle des droits de l'homme, 26e année, n. ${ }^{\circ} 101$, 1er janv. 2015, pp. 89-113.

Prebensen, S.C.: «Importance of the national courts' having duly considered the Convention matter», Human rights law journal (HRLJ), vol. 36, no. 7-12, 30 Dec. 2016, pp. 367-372.

RodríGuez, A.: «La mayor protección interna de los derechos de la convención europea de derechos humanos y el impacto del margen de apreciación nacional», Revista de derecho político, n. ${ }^{\circ}$ 93, 2015, pp. 75-102.

SÁnchez Molina, P.: «Margen de apreciación nacional (en los sistemas de protección internacional de los derechos humanos)», Eunomía: Revista en Cultura de la Legalidad, n. ${ }^{\circ}$ 9, 2015, pp. 224-231.

SPANO, R.: «Universality or diversity of human rights?: Strasbourg in the age of subsidiarity», Human rights law review, vol. 14, no. 3, 2014, pp. 487-502. 
SUdRE, F.: «La mystification du «consensus» européen», La Semaine juridique: édition générale (JCP), 89e année, n. ${ }^{\circ}$ 50, déc. 2015, pp. 2293-2299.

Tsereteli, N. (ed.): «The margin of appreciation in Europe and beyond: special section [collection of articles]», The international journal of human rights, vol. 20, no. 8, 2016, pp. 1055-1131.

Voland, T.: «Advisory opinions of the European Court of Human Rights: unbalancing the system of human rights protection in Europe?», Human rights law review, vol. 17, no. 1, 2017, pp. 73-95.

\section{DIÁLOGO JURISPRUDENCIAL}

\section{A nivel europeo en general y con el Tribunal de Justicia de la Unión Europea}

Libros y capítulos de libro:

Bustos Gisbert, R.: «El diálogo entre el Tribunal de Justicia y el Tribunal Europeo de Derechos Humanos en la construcción de un sistema europeo de defensa de los derechos fundamentales», Gómez Martín, V. (et alii), (Dirs.), Garantías constitucionales y Derecho penal europeo, Marcial Pons Ediciones Jurídicas y Sociales, Madrid, 2012, pp. 169-178.

- «Tribunal de Justicia y Tribunal Europeo de Derechos Humanos: una relación de enriquecimiento mutuo en la construcción de un sistema europeo para la protección de los derechos», García Roca, F. J., y Fernández Sánchez, P. A. (Coords.), Integración europea a través de derechos fundamentales: de un sistema binario a otro integrado, Centro de Estudios Políticos y Constitucionales, Madrid, 2009, pp. 147-168.

Burgorgue-LARSEN, L.: «La formación de un derecho constitucional europeo a través del diálogo judicial», Ferrer MaC-Gregor, E. y Herrera García, A.: Diálogo jurisprudencial en derechos humanos entre tribunales constitucionales y cortes internacionales: in memoriam Jorge Carpizo, generador incansable de diálogos, Tirant Lo Blanch, 2013, pp. 1057-1088.

«La formación de un derecho constitucional europeo a través del dialogo judicial», SAIZ Arnaiz, A. y Ferrer MaC-Gregor, E. (coords): Control de convencionalidad, interpretación conforme y diálogo jurisprudencial: una visión des de América Latina y Europa, Editorial Porrúa, México, 2012.

Dero-Bugny, D.: Les rapports entre la Cour de justice de l'Union européenne et la Cour européenne des droits de l'homme, Bruylant, Bruxelles, 2015.

JiMENA QUESADA, L.: «La interacción de tribunales supremos europeos: reflexiones en homenaje a Luis López Guerra, juez constitucional europeo», Raimondi, G. (et alii) (eds.): Human rights in a global world: essays in honour of judge Luis López Guerra, Wolf Legal Publishers, Oisterwijk, 2018, pp. 389-404.

Martín y Pérez De Nanclares, J.: «El diálogo judicial entre el TJUE y el TEDH: algo más que el derecho a la última palabra en el triángulo judicial europeo», Tribunal Constitucional y diálogo entre tribunales / XVIII Jornadas de la Asociación de Letrados del Tribunal Constitucional, Centro de Estudios Políticos y Constitucionales, Madrid, 2013, p. 161.

Pastor Ridruejo, J.A.: «Las relaciones entre el Tribunal de la Unión Europea y el Tribunal Europeo de Derechos Humanos tras la entrada en vigor de la Constitución para Europa», Aldecoa LuzÁrraga, F. (coord.): Los Tratados de Roma en su cincuenta aniversario: perspectivas desde la Asociación Española de Profesores de Derecho Internacional y Relaciones Internacionales, Marcial Pons, 2008, pp. 325-332.

Petrova, B.: «Three levels of dialogue in precedent formation at the CJEU and ECtH, Petrova, B., Dzentsiarou, K. (et alii) (Eds.): Human rights law in Europe: the influence, overlaps and contradictions of the EU and the ECHR, Routledge, Oxon, 2014, pp. 73-91. 
Ragone, S.: «Las relaciones de los Tribunales Constitucionales de los Estados miembros con el Tribunal de Justicia y con el Tribunal Europeo de Derechos Humanos: una propuesta de clasificación», Ferrer Mac-Gregor, E. y Herrera García, A.: Diálogo jurisprudencial en derechos bumanos entre tribunales constitucionales y cortes internacionales: in memoriam Jorge Carpizo, generador incansable de diálogos, Tirant Lo Blanch, 2013, pp. 415-446.

SENDEN, H. C. K., Interpretation of fundamental rights in a multilevel legal system: an analysis of the European Court of Human Rights and the Court of Justice of the European Union, Intersentia, Antwerp, Belgium, 2011.

\section{Artículos de revista}

Balaguer Callejón, F., «Los tribunales constitucionales en el proceso de integración europea», Revista de Derecho Constitucional Europeo, n..$^{\circ}$ 7, 2007, pp. 327-378.

Del Valle Gálvez, J. A., Rodríguez Iglesias, G. C.: «El Derecho Comunitario y las relaciones entre el Tribunal de Justicia de las Comunidades Europeas, el Tribunal Europeo de Derechos Humanos y los Tribunales Constitucionales nacionales», Revista de Derecho Comunitario Europeo, n. ${ }^{\circ} 2,1997$, pp. 329-376.

Demuro, G., «Las relaciones entre la Corte de Justicia de las Comunidades Europeas y la Corte Europea de Derechos Humanos», Cuestiones constitucionales: Revista mexicana de Derecho Constitucional, n. ${ }^{\circ} 17,2007$.

Hermida Del Llano, C., «Una salida a los conflictos entre el Tribunal de Estrasburgo y el Tribunal de Luxemburgo", Persona y derecho: Revista de fundamentación de las Instituciones Jurídicas $y$ de Derechos Humanos, n. ${ }^{\circ}$ 63, 2010, pp. 111-135.

Herranz Ballesteros, M., «Los Tribunales de Estrasburgo y Luxemburgo ante la protección de los Derechos fundamentales en supuestos de sustracción internacional de menores», Civitas. Revista española de Derecho Europeo, n. ${ }^{\circ} 44,2012$, pp. 41-60.

López Guerra, L.M.: «El Tribunal Europeo de Derechos Humanos, el Tribunal de Justicia de la UE y «le mouvement nécessaire des choses»», Teoría y realidad constitucional, ISSN 11395583, n. ${ }^{\circ}$ 39, 2017, pp. 163-188.

Martín Y Pérez De Nanclares, J.: «El TJUE como actor de la constitucionalidad en el espacio jurídico europeo: la importancia del diálogo judicial leal con los tribunales constitucionales y con el TEDH», Teoría y realidad constitucional, n. ${ }^{\circ}$ 39, 2017, pp. 235-269.

MuÑoz MaChado, S., «Los tres niveles de garantías de los derechos fundamentales en la Unión Europea: problemas de articulación», Revista de Derecho Comunitario Europeo, n. ${ }^{\circ}$ 50, 2015, pp. 195-230.

Ragone, S.: «Las relaciones de los Tribunales Constitucionales de los Estados miembros con el Tribunal de Justicia y con el Tribunal Europeo de Derechos Humanos: una propuesta de clasificación», Revista de Derecho Constitucional Europeo, n. ${ }^{\circ} 16,2011$, pp. 53-90.

Repetto, G., «El argumento comparado en la jurisprudencia de los Tribunales de Estrasburgo y Luxemburgo: el pluralismo de los derechos fundamentales en el orden constitucional europeo», Revista de Derecho Constitucional Europeo, n. . 20, 2013, pp. 277-236.

Ruiz Miguel, C.: «Los derechos fundamentales en la encrucijada entre el Tribunal Europeo de Derechos Humanos y el Tribunal de Justicia de la Unión», Anuario español de derecho internacional, n. ${ }^{\circ} 34,2018$, pp. 641-673.

Sanz Caballero, S., «Interferencias en el Derecho Comunitario y el Convenio Europeo de Derechos Humanos: Luxemburgo versus Estrasburgo, ¿quién es la última instancia de los derechos fundamentales en Europa?» Revista de Derecho Comunitario Europeo, n. ${ }^{\circ}$ 17, 2004, pp. 117-160.

Tenorio SÁnchez, P. J., «Diálogo entre tribunales y protección de los derechos fundamentales en el ámbito europeo», Revista General de Derecho Europeo, n. ${ }^{\circ}$ 31, 2013. 


\section{Con tribunales nacionales}

Libros y capítulos de libro

Alcácer Guirao, R., Beladiez Rojo, M, y SÁNchez Tomás, J.M. (coords): Conflicto y diálogo con Europa: las condenas a España del Tribunal Europeo de Derechos Humanos, Civitas-Thomson Reuters, Cizur Menor, 2013.

Elvira Perales, A.: «Tribunal Constitucional y Tribunal Europeo de Derechos Humanos», Pérez Tremps, P. (coord.): La Reforma del Tribunal Constitucional: actas del V Congreso de la Asociación de Constitucionalistas de España, Tirant lo Blanch, Valencia, 2007, pp. 513-524.

European Court of Human Rights: Dialogue between judges: proceedings of the seminar 31 January 2014: implementation of the judgments of the European Court of Human Rights: a shared judicial responsibility?, Council of Europe, Strasbourg, 2014 ${ }^{10}$.

Dialogue between judges: what are the limits to the evolutive interpretation of the Convention?, Council of Europe, Strasbourg, 2011'11.

Dialogue between judges: the Convention is yours, Council of Europe, Strasbourg, $2010^{12}$.

García Roca, F.J.: «El diálogo entre el Tribunal de Derechos Humanos, los Tribunales Constitucionales y otros órganos jurisdiccionales en el espacio convencional europeo», FerRer Mac Gregor, E. y Herrera García, A.: Diálogo jurisprudencial en derechos humanos entre tribunales constitucionales y cortes internacionales: in memoriam Jorge Carpizo, generador incansable de diálogos, Tirant Lo Blanch, 2013, pp. 219-242.

GLAs, L. R.: The theory, potential and practice of procedural dialogue in the European Convention on Human Rights system, Intersentia, Cambridge; Antwerp; Portland, 2016.

Jimena Quesada, L.: «El diálogo entre el Tribunal Constitucional y el Tribunal Europeo de Derechos Humanos: a propósito del control de convencionalidad», Ferrer Mac Gregor, E. y Herrera García, A.: Diálogo jurisprudencial en derechos bumanos entre tribunales constitucionales y cortes internacionales: in memoriam Jorge Carpizo, generador incansable de diálogos, Tirant Lo Blanch, 2013, pp. 1057-1088.

- Jurisdicción nacional y control de convencionalidad: a propósito del diálogo judicial global y de la tutela multinivel de derechos, Aranzadi, Cizur Menor, 2013.

López Guerra, L.M.: «El diálogo entre el Tribunal Europeo de Derechos Humanos y los tribunales españoles. Coincidencias y divergencias», Gimeno SENDRA, J.V. y Regueiro García, M.T.: Nuevas tendencias en la interpretación de los derechos fundamentales, Universitas, pp. 21-44.

MøSE, E.: «The ECtHR's use of decisions of domestic courts from States not involved in the case and other Council of Europe bodies», MüLlER, A. (ed.): Judicial dialogue and buman rights, Cambridge University Press, Cambridge, 2017, pp. 410-418.

O'BoyLe, M: «The role of dialogue in the relationship between the European Court of Human Rights and national courts», HAECK, Y. (et alii) (ed.): The realisation of human rights: when theory meets practice: studies in honour of Leo Zwaak, Intersentia, Cambridge; Antwerp; Portland, 2013, pp. 91-105.

Pérez Sola, N.: La defensa convencional de los derechos en España: ¿es posible el diálogo entre tribunales?, Instituto Nacional de Administración Pública, Madrid, 2015.

Ripol Carulla, S. (dir.); Ugartemendia Eceizabarrena, J.I. (coord.): España ante los tribunales internacionales europeos: cuestiones de política judicial, Instituto Vasco de Administración Pública, Bilbao, 2008.

10 Versiones en inglés y francés.

11 Versiones en inglés y francés.

12 Versiones en inglés y francés. 
RinCEANU, J.: «Judicial dialogue between the European Court of Human Rights and national supreme courts», SPINELLIS, C.D. (et alii) (eds.): Europe in crisis: crime, criminal justice, and the way forward: essays in honour of Nestor Courakis = Festschrift für Nestor Courakis = Mélanges offerts à Nestor Courakis. Volume II, Essays in English, German, French, and Italian, Ant. N. Sakkoulas, Athens, 2017, pp. 1029-1041.

SaIz Arnaiz, A.: «Tribunal Constitucional y Tribunal Europeo de Derechos Humanos: las razones para el diálogo», Tribunal Constitucional y diálogo entre tribunales / XVIII Jornadas de la Asociación de Letrados del Tribunal Constitucional, Centro de Estudios Políticos y Constitucionales, Madrid, 2013, p. 131.

Thomassen, W.: «The vital relationship between the European Court of Human Rights and national courts», Flogartis, S., Zwart, and T. Fraser, J. (eds.): The European Court of Human Rights and its discontents: turning criticism into strength, Edward Elgar, Cheltenham, 2013, pp. 96-103.

Ulfstein, G.: «The European Court of Human Rights And National Courts: A Constitutional Relationship?», Mjöll Arnardóttir, O. and Buyse, A (ed.): Shifting centres of gravity in human rights protection: rethinking relations between the ECHR, EU and national legal orders, Routledge, Abingdon; Oxon; New York, 2016, pp. 46-58.

\section{Artículos de revistas}

Biavati, P: «La protección de los Derechos Humanos en Europa: relaciones entre los Tribunales supranacionales y los Tribunales Constitucionales de los Estados», Revista General de Derecho Procesal, n. ${ }^{\circ} 28,2012$.

CARrillo, M.: «Procedimiento constitucional y convergencia jurisdiccional en la protección de derechos», Ius fugit: Revista interdisciplinar de estudios bistórico-jurídicos, n. ${ }^{\circ}$ 20, 2017, pp. 47-77.

Dzehtsiarou, K. and O'meara, N: «Advisory jurisdiction and the European Court of Human Rights: a magic bullet for dialogue and docket-control?», Legal studies, 2014, pp. 1-25.

García Roca, F.J.: «El diálogo entre el Tribunal Europeo de Derechos Humanos y los tribunales constitucionales en la construcción de un orden público europeo», Teoría y realidad constitucional, n. ${ }^{\circ} 30,2012$, pp. 183-224.

GLAS, L.R.: «Dialogue in the European Convention on Human Rights system: inspiration, added-value and means = Dialogue dans le système de la Convention européenne des droits de l'homme : origines, valeur ajoutée et moyens», Journal européen des droits de l'bomme = European journal of human rights, $\mathrm{n}^{\circ} 3$, juin 2015, pp. 247-277.

Jimena Quesada, L.: «El diálogo entre el Tribunal Constitucional y el Tribunal Europeo de Derechos Humanos: a propósito del control de convencionalidad», Revista europea de derechos fundamentales, $\mathrm{n}^{\circ}$ 15, 2010, pp. 41-74.

KonDYLIS, V.: «Formes de coopération et de dialogue des juges nationaux avec les juges européens: de l'»antidosis» des qualités à la «circumincession»», Annuaire international des droits de l'homme, vol. 9, 2015-2016, pp. 369-398.

López Guerra, L.: «Dialogues between the Strasbourg Court and national courts», Müller, A. (ed.): Judicial dialogue and buman rights, Cambridge University Press, Cambridge, 2017, pp. 401-409.

«El diálogo entre el Tribunal Europeo de Derechos Humanos y los tribunales españoles. Coincidencias y divergencias», Teoría y realidad constitucional, n. ${ }^{\circ} 32$, 2013, pp. 139-158.

Ripol Carulla, S: «Un nuevo marco de relación entre el Tribunal Constitucional y el Tribunal Europeo de Derechos Humanos», Revista española de derecho internacional, Vol. 66, n. ${ }^{\circ}$, 2014, pp. 11-53. 
RozaKis, C.: «The dialogue between the European Court of Human Rights and national courts», Annuaire international des droits de l'homme, vol. 9 (2015-2016), 2017, pp. 81-84.

\section{Con la Corte Interamericana}

Libros y capítulos de libro

Cano Palomares, G.: «Diálogo entre jurisdicciones supranacionales de derechos humanos: el Tribunal Europeo de Derechos Humanos y la Corte Interamericana de Derechos Humanos», Revenga Sánchez, M. y Cuenca Gómez, P. (eds.): El tiempo de los derechos: los derechos humanos en el siglo XXI, Dykinson, Madrid 2015, pp.47-71.

DíAz Crego, M.: «El impacto de la jurisprudencia de la Corte Interamericana sobre el Tribunal Europeo de Derechos Humanos», Derecho PUCP: Revista de la Facultad de Derecho, n. ${ }^{\circ} 75$, 2015, pp. 31-56.

García Roca, F.J.; Fernández Sánchez, P.A; Santolaya Machetti, P. y Raúl Canosa Usera (Coords.): El Diálogo entre los Sistemas Europeo y Americano de Derechos Humanos, Civitas-Thomson Reuters, Cizur Menor, 2012.

Garro Vargas, A.: «La influencia del Tribunal Europeo de Derechos Humanos en el ejercicio de la función consultiva de la Corte Interamericana de Derechos Humanos», Ferrer MAC Gregor, E. y Herrera García, A.: Diálogo jurisprudencial en derechos bumanos entre tribunales constitucionales y cortes internacionales: in memoriam Jorge Carpizo, generador incansable de diálogos, Tirant Lo Blanch, 2013, pp. 1147-1182.

Kai Амвos, M. L. B.: «Tribunal Europeo de Derechos Humanos y Corte Interamericana de Derechos Humanos: ¿Tribunal tímido vs. Tribunal audaz?», Ferrer MaC Gregor, E. y HerRERA GARCÍA, A.: Diálogo jurisprudencial en derechos humanos entre tribunales constitucionales y cortes internacionales: in memoriam Jorge Carpizo, generador incansable de diálogos, Tirant Lo Blanch, 2013, pp. 1057-1088.

López Guerra, L. y SaIz Arnáiz, A. (ed.): Los sistemas interamericano y europeo de protección de los derechos bumanos: una introducción desde la perspectiva del diálogo entre tribunales, Palestra, Lima, 2015.

Tribunal Europeo de Derechos Humanos: Diálogo transatlántico: selección de jurisprudencia del Tribunal Europeo y la Corte Interamericana de Derechos Humanos, Wolf Legal Publishers (WLP), Oisterwijk, 2015.

Artículos de revistas

Camarillo Govea, L.A: «Convergencias y Divergencias entre los Sistemas Europeo e Interamericano de Derechos Humanos», Revista Prolegómenos. Derechos y Valores de la Facultad de Derecho, Vol. 19, n. ${ }^{\circ}$ 37, 2016, pp. 67-84.

Garro Vargas, A.: «La influencia del Tribunal Europeo de Derechos Humanos en el ejercicio de la función consultiva de la Corte Interamericana de Derechos Humanos», Cuestiones constitucionales: revista mexicana de derecho constitucional, $\mathbf{n} .{ }^{\circ} 20,2009$.

Groppi, T. y Lecis Cocco-Ortu, A.M.: «Las referencias recíprocas entre el Tribunal Europeo y la Corte Interamericana de Derechos Humanos: ¿de la influencia al diálogo?», Revista de derecho politico, n. ${ }^{\circ} 91,2014$, pp. 185-230.

Liakopoulos, D.: «Interactions between European Court of Human Rights and Private International Law of European Union», Cuadernos de derecho transnacional, Vol. 10, n. ${ }^{\circ}$, 2018, pp. 248-305. 


\section{Con otras instituciones}

Libros y capítulos de libro

Jimena Quesada, L.: «Las sinergias entre el Tribunal Europeo de Derechos Humanos y el Comité Europeo de Derechos Sociales: reflexiones sobre relaciones institucionales, informales y humanas en homenaje a Josep Casadevall», López Guerra, L. (et alii) (Coords): El Tribunal Europeo de Derechos Humanos: una visión desde dentro: en homenaje al Juez Josep Casadevall, Tirant lo Blanch, Valencia, 2015, pp.119-133.

Kovács, P.: «Références à la jurisprudence de la Cour européenne des droits de l'homme et de la Cour interaméricaine des droits de l'homme dans la pratique de la Cour pénal internationale», Coussirat-Coustère, V. (dir.): Réciprocité et universalité : sources et régimes du droit international des droits de l'homme: Mélanges en l'bonneur du Professeur Emmanuel Decaux, Pedone, Paris 2017, pp. 245-261.

Pastor Vilanova, P.: «L'improbable dialogue entre la Cour européenne des droits de l'homme et la Cour suprême des Etats-Unis», RAIMONDI, G. (et alii) (eds): Human rights in a global world: essays in honour of judge Luis López Guerra, Wolf Legal Publishers, Oisterwijk, 2018, pp. 441-454.

SaIz Arnaiz, A.: «Estrasburgo en Buenos Aires: citas irrelevantes y omisiones sobresalientes de la doctrina del Tribunal Europeo de Derechos Humanos para justificar el incumplimiento por la Corte Suprema de Justicia de la Nación Argentina de una sentencia de la Corte Interamericana», RAIMONDI, G. (et alii) (Eds): Human rights in a global world: essays in honour of judge Luis López Guerra, Wolf Legal Publishers, Oisterwijk 2018, pp. 455-482.

Ubeda de Torres, A.: «El diálogo entre la Comisión de Venecia y el Tribunal Europeo de Derechos Humanos", RaImONDI, G. (et alii) (Eds): Human rights in a global world: essays in honour of judge Luis López Guerra, Wolf Legal Publishers, Oisterwijk, 2018, pp. 499-513.

\section{Artículos de revistas}

Chatton, G.T.: «La armonización de las prácticas jurisprudenciales del Tribunal Europeo de Derechos Humanos y del Comité Europeo de Derechos Sociales: una evolución discreta», Revista de Derecho Político, n. ${ }^{\circ}$ 73, Septiembre-Diciembre 2008, pp. 273-310.

\section{REVISTAS JURÍDICAS QUE INCLUYEN PERIÓDICAMENTE CRÓNICAS DE JURISPRUDENCIA DEL TEDH.}

A continuación, algunas de las revistas jurídicas en las que se pueden encontrar periódicamente secciones dedicadas a comentarios doctrinales sobre la jurisprudencia del TEDH:

Anuario de Derecho Público, Universidad Diego Portales. En la sección anual titulada «Jurisprudencia de la Gran Sala del Tribunal Europeo de Derechos Humanos».

European public law, Kluwer Law International. Desde 1997, en la sección titulada «European Court of Human Rights».

Human Rights Law Journal, N.P. Engel. En la sección titulada «DeCISIONS and reports»- European Court of Human Rights».

Revista de Administración Pública, Centro de Estudios Políticos y Constitucionales. Desde 2005, n. ${ }^{\circ} 168$, en la sección titulada «Notas de jurisprudencia del Tribunal Europeo de Derechos Humanos» (autor: BouAZZA ARIÑo, O.). 
Revista de Derecho Comunitario Europeo, Centro de Estudios Políticos y Constitucionales. En la sección titulada «Crónica de jurisprudencia Del Tribunal Europeo De Derechos Humanos» (Autora: Cabrera Martín, M.).

Revista de justicia administrativa, Lex Nova. Hasta 2013, en la sección titulada «Derechos fundamentales y libertades públicas».

Revista española de derecho administrativo, Aranzadi. Desde el 2014 (n. $\left.{ }^{\circ} 164\right)$, en la sección titulada «derechos fundamentales».

Revista española de derecho europeo, Aranzadi. En la sección titulada «Comentarios de jurisprudencia».

Revista europea de derechos fundamentales, Universidad Rey Juan Carlos: Instituto de Derecho Público. En la sección titulada «Crónica de la Jurisprudencia del Tribunal Europeo de Derechos Humanos».

Revista española de derecho financiero, Civitas. Desde 2007, en la sección «Jurisprudencia del Tribunal Europeo de Derechos Humanos» (Autora: MarTínez MuÑoz, Y.).

Title: European Court of Human Rights: mutations and challenges. Bibliographic Repertory (2007-2018)

Abstract: The present repertoire gathers a selection of various doctrinal contributions (books, chapters and papers) on the European Court of Human Rights which have been published in the last decade. They are a reflection of some of the challenges that the Court has lately faced, and they tackle issues as its organization, the procedure and access to its jurisdiction, the enforcement of its judgments and their impact, its dialogue with other courts, and some principles or concepts guiding its practice, such as the margin of appreciation, consensus and subsidiarity.

Resumen: El presente repertorio of rece una selección de obras bibliográficas (libros, capítulos de libro y artículos de revistas) sobre el Tribunal Europeo de Derechos Humanos publicadas desde hace algo más de una década. Estos trabajos reflejan algunos de los desafíos a los que se ha enfrentado el Tribunal en los últimos tiempos, y se centran en temas como la organización de la institución y el procedimiento que ante la misma se sustancia, la ejecución y los efectos de sus sentencias, su interacción o diálogo con otros tribunales, o ciertos principios y conceptos que orientan su actuación, como son la subsidiariedad, el consenso y el margen de apreciación nacional.

Key words: European Court of Human Rights, reform, procedure, remedial measures, enforcement of judgments, impact, margin of appreciation, consensus, subsidiarity, judicial dialogue, repertory.

Palabras clave: Tribunal Europeo de Derechos Humanos, reforma, procedimiento, reparación, ejecución de sentencias, impacto, margen de apreciación nacional, consenso, subsidiariedad, diálogo judicial, repertorio.

FECHA DE RECEPCIÓN: 16.07.2018

FECHA DE ACEPTACIÓN: 13.09.2018 
